\title{
Perirhinal and Parahippocampal Cortices Differentially Contribute to Later Recollection of Object- and Scene-Related Event Details
}

\author{
Bernhard P. Staresina, ${ }^{1}$ Katherine D. Duncan, ${ }^{2}$ and Lila Davachi ${ }^{2,3}$ \\ ${ }^{1}$ Medical Research Council Cognition and Brain Sciences Unit, Cambridge CB2 7EF, United Kingdom, and ${ }^{2}$ Department of Psychology and ${ }^{3}$ Center for \\ Neural Science, New York University, New York, New York 10003
}

How the different elements of our experiences are encoded into episodic memories has remained one of the major questions in memory research. Although the pivotal role of the medial temporal lobe as a whole for memory formation is well established, much controversy surrounds the precise contributions of the subregions in the medial temporal lobe cortex (MTLC), most notably the perirhinal cortex $(\mathrm{PrC})$ and the parahippocampal cortex $(\mathrm{PhC})$. Although one prominent view links $\mathrm{PrC}$ processes with familiarity-based memory and $\mathrm{PhC}$ with recollection, an alternative organizing principle is the representational domain critical for successful memory performance (e.g., object- versus scene-related information). In this functional magnetic resonance imaging study, we directly compared successful source encoding during object versus scene imagery, holding perceptual input constant across the two representational domains. Although the hippocampus contributed to associative encoding of both object and scene information, our results revealed a clear double dissociation between $\mathrm{PrC}$ and $\mathrm{PhC}$ for object- versus scene-related source encoding. In particular, PrC, but not $\mathrm{PhC}$, encoding activation predicted later source memory for the object imagery task, whereas $\mathrm{PhC}$, but not $\mathrm{PrC}$, encoding activation predicted later source memory for the scene imagery task. Interestingly, the transitional zone between $\mathrm{PrC}$ and posterior PhC contributed to both object and scene source encoding, possibly reflecting a gradient in domain preference along MTLC. In sum, these results strongly point to representational domain as a key factor determining the involvement of different MTLC subregions during successful episodic memory formation.

\section{Introduction}

How are our rich everyday life experiences encoded into lasting memory traces? Although the pivotal role of the medial temporal lobe (MTL) for episodic memory is well established, much controversy still surrounds the precise contributions of different MTL subregions, most notably the hippocampus and the adjacent parahippocampal gyrus (PhG), including perirhinal cortex $(\mathrm{PrC})$ and parahippocampal cortex $(\mathrm{PhC}$ ) (for review, see Squire et al., 2004; Davachi, 2006; Eichenbaum et al., 2007).

One fruitful approach in delineating the roles of MTL subregions has focused on the different phenomenological states contributing to recognition memory (i.e., stimulus familiarity and recollection of associated event details) (Mandler, 1980; Jacoby, 1991; Yonelinas, 2002). Specifically, it has been suggested that, whereas the hippocampus supports associative or contextual recollection, PrC supports familiarity-based item memory (Brown and Aggleton, 2001; Diana et al., 2007; Eichenbaum et al., 2007;

\footnotetext{
Received Sept. 23, 2010; revised Feb. 10, 2011; accepted Feb. 23, 2011.

Author contributions: B.P.S., K.D.D., and L.D. designed research; B.P.S. and K.D.D. performed research; B.P.S. and K.D.D. contributed unpublished reagents/analytic tools; B.P.S. analyzed data; B.P.S. and L.D. wrote the paper.

This work was supported by a Sir Henry Wellcome Postdoctoral Fellowship (B.P.S.) and National Institute of Mental Health Grant R01 MH074692 (L.D.). We thank Rik Henson for his helpful comments.

This article is freely available online through the J Neurosci Open Choice option.

Correspondence should be addressed to Dr. Bernhard P. Staresina, Medical Research Council Cognition and Brain Sciences Unit, 15 Chaucer Road, Cambridge CB2 7EF, UK. E-mail: bernhard.staresina@mrc-cbu.cam.ac.uk.

DOI:10.1523/JNEUROSCI.4978-10.2011

Copyright $\odot 2011$ the authors $\quad 0270-6474 / 11 / 318739-09 \$ 15.00 / 0$
}

Mayes et al., 2007). Support for this distinction has come from functional magnetic resonance imaging (fMRI) studies showing that encoding activation in $\mathrm{PrC}$ correlates with later memory for the studied item (e.g., a word or an object), whereas hippocampal and $\mathrm{PhC}$ encoding activation correlates with source memory for spatial/contextual episodic details (Davachi et al., 2003; Ranganath et al., 2004; Kensinger and Schacter, 2006; Dougal et al., 2007).

An alternative account of MTL function is based on the notion that different stimulus representations/domains are differentially processed by distinct MTL subregions ("representational account”) (Davachi, 2006). Evidence for domain-based heterogeneity in the MTL has been provided by lesion studies in rats and monkeys (Meunier et al., 1993; Eacott et al., 1994; Wan et al., 2001; Alvarado and Bachevalier, 2005) (for review, see Eacott and Gaffan, 2005) as well as neuropsychological (Lee at al., 2005; Taylor et al., 2007) and functional imaging (Pihlajamäki et al., 2004; Awipi and Davachi, 2008; Litman et al., 2009) studies in humans. Specifically, PrC and PhC have been shown to be differentially responsive to object versus scene stimuli, respectively, consistent with neuroanatomical studies in primates revealing differential amounts of input from object (ventral)- and scenespecific (dorsal) processing streams to these regions (Suzuki and Amaral, 1994).

The current study was designed to directly test whether episodic encoding of information from different representational domains is supported by different MTL subregions. To this end, we used a 
source encoding paradigm in which the tobe-remembered source information (the task performed during the study episode) was either object or scene related, with the perceptual input being identical (see Fig. 1). If the representational domain of the criterial event information indeed modulates the involvement of different MTL subregions, one would expect successful encoding of object-related source details to be supported by $\mathrm{PrC}$, whereas successful encoding of scene-related source details might be supported by $\mathrm{PhC}$.

\section{Materials and Methods}

Subjects. Twenty-two (nine female) righthanded native English speakers with normal or corrected-to-normal vision participated in the experiment (mean age, 24 years; range, 18-32). Informed consent was obtained in a manner approved by the Institutional Review Board at New York University, and subjects were paid for their participation. Three subjects were excluded from all analyses because of early termination of the experiment.

Stimuli. The stimulus set consisted of 336 English adjectives, 224 of which served as study items and 112 of which served as lures during a recognition memory test. To select these adjectives, we had five subjects rate the difficulty of imagining a set of 496 adjectives as either an object or a scene (between-subjects design) on a five-point scale (see below). The rating for each adjective was then $z$-transformed within subjects, and a difference score for object versus scene imagery was calculated across subjects. The 336 adjectives with the smallest difference scores, meaning adjectives that were most similarly imaginable for objects and scenes across subjects, were included in the final stimulus list (mean difference score, 0.39 ). Thus, we controlled for imagery difficulty across object and scene trials. For counterbalancing purposes, these 336 adjectives were divided into three lists of 112 adjectives each, and the assignment of "object image," "scene image," or "test lure" to each adjective was rotated across subjects.

Procedure. For each scanned $5 \mathrm{~s}$ encoding trial, subjects were presented with an adjective in black letters on a white background along with the cue "OBJECT" or "SCENE" above the adjective (Fig. 1). The order of object and scene trials was randomized. After $3.5 \mathrm{~s}$, the adjective and cue disappeared and a rating screen was displayed for the remaining $1.5 \mathrm{~s}$, showing the cue "RATING" followed by the options " $0-1-2-3-4$." Subjects were instructed to vividly imagine an object or a scene (depending on the cue) that could be described by the adjective for the entire $3.5 \mathrm{~s}$ and to indicate their success in the remaining $1.5 \mathrm{~s}$ via a button press. Trials for which no response was given within the $1.5 \mathrm{~s}$ answering period were excluded from all analyses (average, $3 \pm 1 \%$ across subjects). We encouraged subjects to focus only on objects or scenes, respectively, by instructing, "... For example, if the adjective is 'dirty' and the cue is 'object,' you should vividly imagine a dirty object, such as a full garbage can. Conversely, if the cue is 'scene,' you should vividly imagine a dirty scene or place, such as a garbage dump or a messy room. It is crucial that, for object trials, you only focus on imagining an object, without embedding it into a scene image. For scene trials, please avoid incorporating people into the scene if at all possible. Instead, focus on imagining a vivid spatial environment that describes the adjective." For the imagery rating, the instructions were as follows: "To rate the quality of your mental image, decide where it would fit on a scale from 0 to 4 , where 0 indicates the failure to come up with a vivid mental image and 4 indicates that you successfully conjured up a vivid and detailed mental image."

After the encoding session, subjects were given an unscanned and self-paced surprise recognition memory test (Fig. 1), consisting of all 224 previously presented adjectives as well as 112 novel adjectives (lures). First, subjects were instructed to indicate whether the adjective was old (presented during the encoding session) or new (not presented during the encoding session). For adjectives endorsed as old, subjects were then prompted to indicate the imagery task with which they had encountered the adjective (object or scene imagery) including confidence ratings. Answer options were "sure object"- "unsure object"—"?"- "unsure scene"- "sure scene." Note that subjects were not forced to guess but could indicate they do not know the corresponding source ("?" response). This testing protocol was used to sort the scanned encoding trials based on successful and unsuccessful source encoding as a function of representational domain (object vs scene trials). Successful and unsuccessful source encoding were operationalized by considering both accuracy and confidence during the subsequent source test: Trials leading to correct-"sure" responses were defined as successful source encoding and trials leading to incorrect-"unsure" or "?" responses were defined as unsuccessful source encoding. Correct-unsure and incorrect-sure responses were not included in the analysis because of the ambiguous combination of accuracy and confidence. However, the results reported below remain the same when segmenting trials into "correct" versus "?/incorrect" source memory, without taking confidence ratings into account. The item recognition component allowed us to isolate source memory by including only trials with successful item memory (i.e., "hits"), but, since we are interested in differential source encoding effects 
across MTL regions, trials leading to unsuccessful item memory (i.e., "misses") are not considered further herein. Thus, to reiterate, our main conditions of interest were (1) successful source encoding, object trials; (2) unsuccessful source encoding, object trials; (3) successful source encoding, scene trials; and (4) unsuccessful source encoding, scene trials, together representing a 2 (successful vs unsuccessful source encoding) $\times$ 2 (object vs scene trials) factorial design.

MRI scanning and data analysis. Scanning was performed on a 3T Siemens Allegra MRI system using a whole-head coil. Functional data were acquired using a gradient-echo, echo-planar pulse sequence (repetition time, $1500 \mathrm{~ms}$; echo time, $30 \mathrm{~ms} ; 27$ slices oriented perpendicular to the hippocampal axis; $3 \times 3 \times 3 \mathrm{~mm}$ voxel size; $0.6 \mathrm{~mm}$ interslice gap; 317 volume acquisitions per run). The first eight volumes of each run were discarded to allow for magnetic field stabilization. High-resolution T1-weighted (magnetization-prepared rapid acquisition gradient echo) images were collected for anatomical visualization. Foam padding was used to minimize head motion. Visual stimuli were projected onto a screen that was viewed through a mirror, and responses were collected with a magnet-compatible button box placed under the subject's left hand.

The scanned encoding portion of the experiment was divided into eight runs, each encoding run consisting of 28 trials. Stimuli were presented in a slow event-related fashion, with each encoding trial followed by a sensorimotor baseline task ["arrows task" (Stark and Squire, 2001)]. Here, a sequence of arrows that randomly pointed to the left or to the right for $1 \mathrm{~s}$ was presented for the length of a baseline trial (10-13 s), and subjects pressed the left middle finger key if the arrow pointed to the left and the left index finger key if it pointed to the right.

Data were analyzed using SPM5 (Wellcome Department of Cognitive Neurology, London, UK). During preprocessing, images were corrected for differences in slice acquisition timing, followed by motion correction across all runs. Structural images were coregistered to mean functional images resulting from motion correction. For the standard whole-brain analysis (see below), preprocessing further included spatial normalization of the coregistered structural image to a $\mathrm{T} 1$ template provided in SPM5. The resulting normalization parameters were then applied to the functional images, followed by spatial smoothing with a $6 \mathrm{~mm}$ full-width, half-maximum isotropic Gaussian kernel.

For statistical analyses, we used two separate analysis approaches: First, to assess the pattern of activation across the $\mathrm{PhG}$ while taking each subject's MTL anatomy into account, we used a hand-drawn anatomical region of interest (ROI) approach. Here, data were not normalized or smoothed during preprocessing. Based on each subject's T1-weighted structural scan, we segmented the PhG into three equidistant portions: an anterior portion (ant-PhG) covering $\mathrm{PrC}$, a posterior portion (post$\mathrm{PhG}$ ) covering $\mathrm{PhC}$, and a mid-PhG portion covering the transition between $\mathrm{PrC}$ and $\mathrm{PhC}$. Anatomical demarcation was performed according to Insausti et al. (1998) and Pruessner et al. (2002), starting at the most anterior portion of the $\mathrm{PrC}$ and ending at the most posterior portion of the PhC at the level of the posterior hippocampus. For each condition of interest, percentage signal change relative to baseline was extracted within the resulting six ROIs (left and right ant-PhG, mid-PhG, post-PhG) via the MarsBaR toolbox (Brett et al., 2002).

Second, to complement the ROI analysis and to query additional MTL regions, we used a standard whole-brain general linear model (GLM) approach. Here, neural activity was modeled via boxcar functions spanning the $3.5 \mathrm{~s}$ imagery period and convolved with a canonical hemodynamic response function together with its first-order temporal derivative. The resulting vectors were then entered as regressors into a fixed-effects GLM together with nuisance regressors modeling run means and scanner drift after concatenating the eight encoding runs. Parameter estimates ( $\beta$-weights) for each condition of interest were derived for each subject and carried forward to a second-level group analysis. Here, individual subjects' $\beta$-weights for the four conditions of interest were entered into a repeated-measures ANOVA, and domain preference during source encoding was assessed via directed contrast and masking analyses (further detailed below).

Additional behavioral study. Given our behavioral results (see below), we conducted a separate behavioral study to directly assess the contribu- tions of recollection and familiarity to source memory decisions in the current paradigm. Eleven (seven female) right-handed native English speakers with normal or corrected-to-normal vision participated in the experiment (mean age, 22 years; range, 19-26). Informed consent was obtained in a manner approved by the Institutional Review Board at New York University, and subjects were paid for their participation. One subject was excluded from all analyses for not responding on $34 \%$ of the trials and only giving imagery ratings of 4 on the remaining trials. The results, however, are unaffected by inclusion or exclusion of that subject. The same adjectives were used as in the main fMRI experiment. The encoding procedures were modified in two ways: first, we reduced the duration of the intervening arrows trials to $4 \mathrm{~s}$. Second, because we did not use lures during retrieval, all 336 adjectives were shown during the encoding session. Importantly, we modified the testing protocol to directly assess recollection and confidence levels for subjects' source memory answers. On being presented with an adjective, subjects had six response options: recollect object- high confidence object-low confidence object-low confidence scene- high confidence scene-recollect scene. Subjects were instructed to use recollection and confidence in the following manner: "Recollection indicates that you recall specific details about the study phase including memory for the mental image that you generated on the study trial or any other details about the study phase that indicates whether you imagined an object or a scene. High and low confidence indicate that you are very sure or moderately sure, respectively, that you imagined an object or a scene, without being able to recollect specific details. Is this distinction clear? Please explain in your own words." As for the main fMRI study, the retrieval portion was self-paced.

\section{Results}

\section{Behavioral results}

First, we assessed whether imagery success during encoding varied as a function of the representational domain by comparing the proportion of object to scene trials for each rating level $(0-4)$. A repeatedmeasures ANOVA with the factors domain (object, scene) and rating $(0,1,2,3,4)$ revealed no main effect of domain $\left(F_{(1,18)}=1.66\right.$; $p>0.20)$ or domain by rating interaction $\left(F_{(4,72)}=1.17 ; p>\right.$ $0.32)$, indicating that, for no rating level, was there a higher proportion of object or scene trials. The overall imagery rating was $2.54( \pm 0.07)$, and as expected, imagery ratings correlated positively with subsequent source memory. In particular, the proportion of successful to unsuccessful source encoding increased stepwise with increasing imagery rating: $52,54,65,72$, and $80 \%$ for ratings $0-4\left(F_{(4,72)}=33.98 ; p<0.001\right)$. However, as there was no interaction between imagery rating and domain on the proportion of successful source encoding $\left(F_{(4,72)}=0.51 ; p>\right.$ 0.72 ) and since our question focuses on differential source encoding effects across representational domains, imagery ratings will not be considered further here.

During the recognition test, 78\% (SEM, 2\%) of all old adjectives were correctly identified as old (hits), whereas 87\% (SEM, $2 \%$ ) of all new adjectives (lures) were correctly identified as new ("correct rejections"). Of all hits, the correct source, namely whether the adjective had been associated with object or scene imagery during encoding, was correctly remembered for $70 \%$ (SEM, 3\%). Performance on item recognition and source tests was comparable for object and scene trials, reflected in the absence of an interaction between domain (object, scene) by memory test performance (item, source) $\left(F_{(1,18)}=2.47 ; p>0.12\right)$. The overall proportion of correct source trials (of all hits) was virtually identical across representational domains: $70.1 \%( \pm 2.5 \%)$ for object trials and $70.2 \%( \pm 3.3 \%)$ for scene trials $\left(t_{(18)}=0.02\right.$; $p>0.97$ ), which was markedly above chance (assuming a chance rate of $50 \%$ when not considering the "?" response option) for both trial types (both $t_{(18)}>6.12 ; p<0.001$ ). Interestingly, when 
considering confidence ratings for correct source decisions, object trials showed a trend of entailing a larger proportion of correct sure responses ( $51 \%$ for object trials vs $46 \%$ for scene trials, taken as the proportion of all item hits; $t_{(18)}=1.97, p=0.06$ ).

In light of the aforementioned phenomenological account (familiarity vs recollection), it is unclear whether the higher proportion of correct sure responses for object source memory is driven by enhanced feelings of familiarity or by recollection of specific details for object trials. We thus conducted a separate behavioral study to directly assess the contributions of recollection and familiarity to source memory decisions (for details, see Materials and Methods). As in the imaging study, there was no difference between object and scene trials with respect to imagery success $\left(F_{(4,36)}=1.11 ; p>0.36\right)$, and overall source accuracy was $\sim 70 \%[69 \%( \pm 4 \%)$ for object trials and $71 \%( \pm 3 \%)$ for scene trials $\left.\left(t_{(9)}=0.49 ; p>0.63\right)\right]$, ensuring comparability between the behavioral and the main fMRI study. Critically, of all memory outcomes, object trials yielded a significantly higher proportion of correct "recollect" responses than scene trials ( $38 \mathrm{vs} 18 \%$; $t_{(9)}=$ 4.48; $p<0.005$ ) (supplemental Fig. S5, available at www. jneurosci.org as supplemental material). The same pattern was obtained when taking the proportion of correct recollect responses from all correct source responses only [i.e., correct recollect/(correct recollect + correct high confidence + correct low confidence); 55 vs $\left.25 \% ; t_{(9)}=4.55 ; p<0.005\right]$ or when taking the proportion of correct recollect responses from correct recollect and correct "high confidence" responses only [correct recollect/ (correct recollect + correct high confidence); 72 vs $50 \% ; t_{(9)}=$ $4.09 ; p<0.005]$. Conversely, correct source memory for scene trials was accompanied by significantly higher levels of familiarity than source memory for object trials, regardless of whether familiarity was calculated as (1) correct low confidence/all responses, (2) correct low confidence/( 1 - correct recollect), or (3) (correct high confidence + correct low confidence $) /(1$ - correct recollect) (all $t_{(9)}>2.65 ; p<0.05$ ), where (2) and (3) are analogous to the "independent- $K$ " procedure (Yonelinas and Jacoby, 1995). Of course, it is possible that successful source memory may also be accompanied by higher levels of familiarity than unsuccessful source memory in general. Nevertheless, the current data strongly suggest that source memory for object trials included high levels of recollection in our paradigm.

\section{Imaging results}

\section{Anatomical ROI analysis}

As a first approach, the peristimulus percentage signal change was extracted for our four conditions of interest (successful vs unsuccessful source encoding for object and scene trials) across each subject's PhG (ant-PhG, mid-PhG, post-PhG) (example shown in Fig. 2). Statistics were calculated on the average of time points 3 and 4 (corresponding to 4.5-6 s) after stimulus onset, which represented the peak time points of the hemodynamic response collapsed across all ROIs and conditions (supplemental Fig. S1A, available at www.jneurosci.org as supplemental material) [note that the pattern of results did not depend on the exact choice of time points included for statistical analysis (supplemental Fig. S1B, available at www. jneurosci.org as supplemental material)].

To assess domain preference across the PhG, we conducted a repeated-measures ANOVA including the factors hemisphere (left, right), region (ant-PhG, mid-PhG, post-PhG), domain (object, scene), and source encoding (successful, unsuccessful). Most critically, results revealed a significant region by domain by source encoding interaction $\left(F_{(2,36)}=13.97 ; p<0.001\right)$. Because this interaction did not further interact with the factor hemisphere $\left(F_{(2,36)}=0.17 ; p>0.84\right)$, data from left and right hemisphere ROIs were pooled for subsequent analyses. To unpack this triple interaction, we next compared source encoding effects for object and scene trials separately for each of the three PhG segments. Results revealed that the ant- $\mathrm{PhG}$ region (covering $\mathrm{PrC}$ ) showed a significant source encoding effect for object trials $\left(t_{(18)}=4.23 ; p<\right.$ $0.001)$, but not for scene trials $\left(t_{(18)}=0.23 ; p>0.47\right)$, whereas the exact inverse pattern was seen in the post-PhG region (covering $\mathrm{PhC}$ ), showing a significant source encoding effect for scene trials $\left(t_{(18)}=3.14 ; p<0.01\right)$, but not for object trials $\left(t_{(18)}=0.16 ; p>\right.$ $0.86)$ (Fig. 2). Finally, the mid-PhG region (covering the transition between $\mathrm{PrC}$ and $\mathrm{PhC}$ ) showed source encoding effects for both object and scene trials (both $t_{(18)}>2.28 ; p<0.05$ ). Note that we used unsmoothed data for the ROI analysis, ruling out the possibility that the mid-PhG pattern might be attributable to spillover from the domain-preferential source encoding effects seen in the anterior and posterior PhG ROIs. The underlying time course data for both hemispheres are presented in supplemental Figure S1 B (available at www.jneurosci.org as supplemental material). In sum, the ROI analysis revealed a striking double dissociation between $\mathrm{PrC}$ and $\mathrm{PhC}$ for successful source encoding during object and scene trials, respectively.

We also conducted the same analysis without the mid-PhG transition zone, segmenting each subject's $\mathrm{PhG}$ only into $\mathrm{PrC}$ and $\mathrm{PhC}$ according to Insausti et al. (1998) and Pruessner et al. (2002) (example shown in supplemental Fig. S2, available at www. jneurosci.org as supplemental material). After the procedure described above, the repeated-measures ANOVA (now with only two levels of the factor region- $\operatorname{PrC}$ and $\mathrm{PhC}$ ) again revealed a significant region by domain by source encoding interaction $\left(F_{(1,18)}=14.25 ; p<0.005\right)$. Because this interaction did not further interact with the factor hemisphere either $\left(F_{(2,36)}=0.99\right.$; $p>0.32)$, data from left and right hemisphere ROIs were pooled for subsequent analyses. Source encoding effects for object and scene trials were then compared separately for the $\mathrm{PrC}$ and $\mathrm{PhC}$ ROIs. Replicating the results reported above, $\operatorname{PrC}$ showed a significant source encoding effect for object trials $\left(t_{(18)}=4.01 ; p<\right.$ $0.001)$, but not for scene trials $\left(t_{(18)}=1.20 ; p>0.23\right)$, whereas $\mathrm{PhC}$ showed a significant source encoding effect for scene trials $\left.t_{(18)}=3.16 ; p<0.01\right)$, but not for object trials $\left(t_{(18)}=0.80 ; p>\right.$ 0.42 ). In sum, our results show that, although a coarse segmentation of the $\mathrm{PhG}$ into $\mathrm{PrC}$ and $\mathrm{PhC}$ reveals strong evidence for domain preference during successful source encoding, the anatomical area of transition between these regions shows significant, albeit less pronounced, source encoding effects for both trial types.

\section{Standard GLM approach}

To complement the ROI analysis and to reveal potential source encoding effects beyond our predefined regions of interest, we used a voxelwise GLM approach. To identify domain preference in a stringent, one-step procedure, we performed contrasts together with exclusive masking. Specifically, to identify MTL regions supporting domain-preferential source encoding for object trials, we set up the contrast "successful $>$ unsuccessful source encoding, object trials" and masked the results exclusively with the contrast "successful > unsuccessful source encoding, scene trials." The inverse contrast/masking procedure was applied to reveal scene-preferential source encoding regions. Statistical parametric maps were initially thresholded at $p<0.001$ (uncorrected) with a minimum of 5 contiguous voxels, and results were corrected for multiple comparisons via small volume correction 


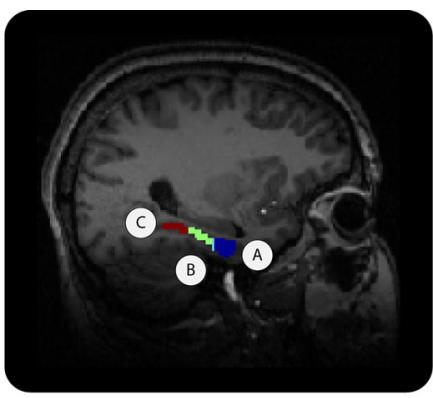

C posterior $\mathrm{PhG}$ parahippocampal cortex $(\mathrm{PhC})$
B mid PhG

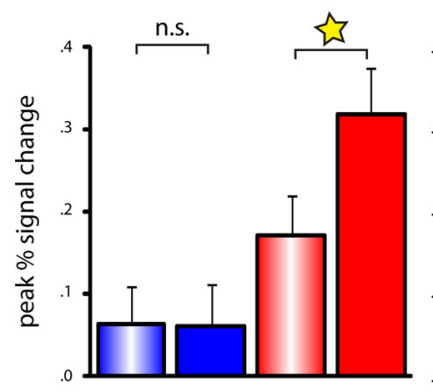

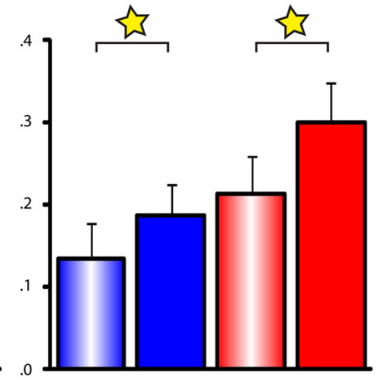
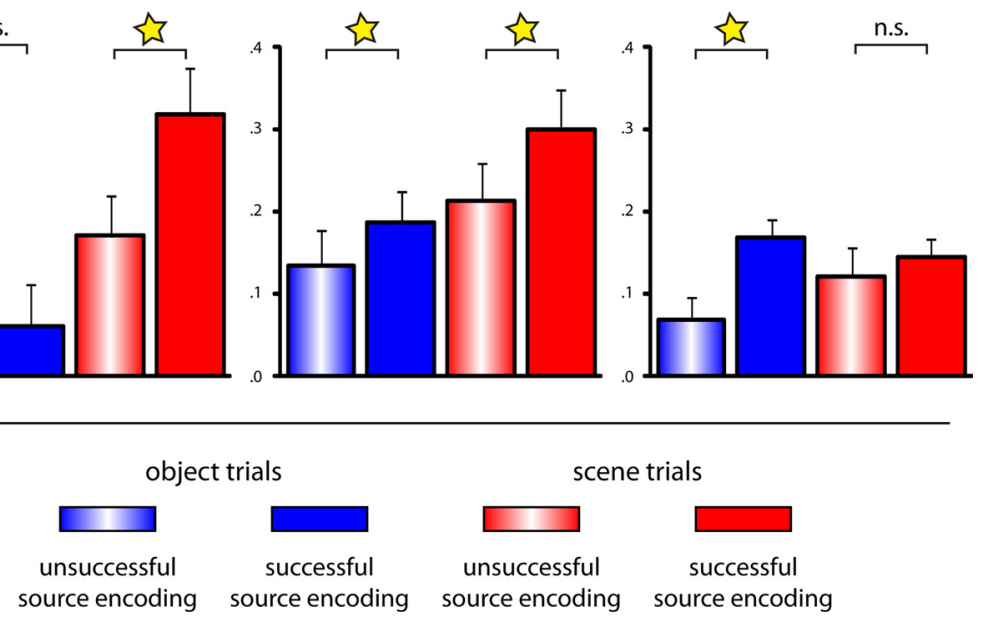

Figure 2. Transition of domain-specific source encoding effects along MTLC revealed via anatomical ROI analyses. Top, Example segmentation of the PhG into anterior $(\boldsymbol{A})$ (covering perirhinal cortex), mid $(\boldsymbol{B})$, and posterior $(\boldsymbol{C})$ (covering parahippocampal cortex) portions. Bottom, Bar graphs representing mean (+SEM) peak percentage signal change for successful (filled bars) and unsuccessful (striped bars) object (blue) and scene (red) source encoding trials. ${ }^{2} p<0.05$; n.s., not significant.

(SVC) (Worsley et al., 1996), using a hand-drawn small volume mask covering the hippocampus and the parahippocampal gyrus bilaterally (1319 voxels) (supplemental Fig. S3, available at www. jneurosci.org as supplemental material). Note that we used a very liberal threshold for the exclusive mask ( $p<0.1$, uncorrected; no extent threshold), rendering the assessment of domain preference more conservative.

First, the only cluster that emerged from the object-preferential source encoding analysis was located in the right $\operatorname{PrC}$ (Fig. 3A) [peak Montreal Neurological Institute (MNI) coordinates: 33, -6, -30], surviving correction for multiple comparisons (SVC) in the MTL volume at $p_{\text {corrected }}<0.05$. Second, the only cluster that emerged from the scene-preferential source encoding analysis was located in the right PhC (Fig. 3A) (peak MNI coordinates: 27, -36, -9), again surviving correction for multiple comparisons in the MTL volume at $p_{\text {corrected }}<0.05$. Thus, the GLM approach accurately mirrored the anatomical ROI results described above, revealing object-preferential source encoding effects in $\operatorname{PrC}$ and scene-preferential source encoding effects in PhC.

To add to the analysis for domain-preferential source encoding effects, we asked whether any other MTL regions would show "domain-general" source encoding effects in our paradigm (i.e., show source encoding effects for both object and scene trials). Domain-general source encoding effects were identified by inclusively masking the contrast "successful $>$ unsuccessful source encoding, object trials" with the contrast "successful $>$ unsuccessful source encoding, scene trials," each thresholded at $p<$
A anterior PhG perirhinal cortex $(\operatorname{PrC})$
$0.001^{1 / 2}$ ( 5 contiguous voxels minimum). Consistent with the results from our ROI analysis, domain-general source encoding effects were seen in bilateral mid-PhG regions (Fig. 3B). Importantly, domaingeneral source encoding effects were also seen in the hippocampus (Fig. $3 B$ ). It is interesting to note that, whereas scene trials generally elicited greater hippocampal engagement (main effect of representational domain; $\left.F_{(1,18)}=5.83, p<0.05\right)$, the source encoding effects (i.e., the difference between successful and unsuccessful source encoding) were equally pronounced for object and scene trials, reflected by a main effect of source encoding $\left(F_{(1,18)}=16.74 ; p<0.001\right)$ in the absence of a domain by source encoding interaction $\left(F_{(1,18)}=0.09 ; p>0.76\right)$. This finding corroborates the proposed role of this region in associative/source binding regardless of representational domains (Cohen and Eichenbaum, 1993; Davachi, 2006; Konkel and Cohen, 2009).

\section{Episodic encoding versus mental imagery} One potential concern about our current paradigm is to what extent the source encoding effects we observed reflect "true" episodic encoding as opposed to merely reflecting successful mental imagery. This question is of particular importance since, behaviorally, imagery success was strongly correlated with later memory performance. To assess whether successful source encoding in $\mathrm{PrC}$ and $\mathrm{PhC}$ is independent of mental imagery, we set up another GLM in which two parametric modulators (Büchel et al., 1998) were entered for object and scene trials as follows: (1) a parametric modulator reflecting the mental imagery success (ranging from 0 to 4 ) and (2) a parametric modulator reflecting the source encoding success (coding "unsuccessful source encoding" as 1 and "successful source encoding" as 2) across trials. The key aspect of this analysis is that, when sequentially entering two parametric modulators, the second modulator gets orthogonalized with respect to the first (i.e., variance common to both modulators will be assigned to the mental imagery modulator). Hence, a parameter estimate for the source encoding modulator greater than zero after orthogonalization reflects a significant contribution to source encoding independent from (or unaccountable by) mental imagery. To statistically assess imagery-independent source encoding effects in $\mathrm{PrC}$ and $\mathrm{PhC}$, the parameter estimates for the orthogonalized source encoding modulator were extracted from the $\mathrm{PrC}$ and $\mathrm{PhC}$ clusters emerging from the GLM analysis above. Critically, we observed the same double dissociation between $\mathrm{PrC}$ and $\mathrm{PhC}$ source encoding effects during object and scene trials. In PrC, the parameter estimate for the orthogonalized source encoding modulator was significantly greater than zero for object trials $\left(t_{(18)}=4.60\right.$; $p<0.001)$ but not for scene trials $\left(t_{(18)}=0.19 ; p>0.85\right)$, whereas in $\mathrm{PhC}$ the estimate was significantly greater than zero for scene trials $\left(t_{(18)}=2.33 ; p<0.05\right)$ but not for object trials $\left(t_{(18)}=-1.60 ; p>0.12\right)$. Again, the double dissociation 
A Domain-Specific Source Encoding

Object Trials Perirhinal Cortex (PrC)
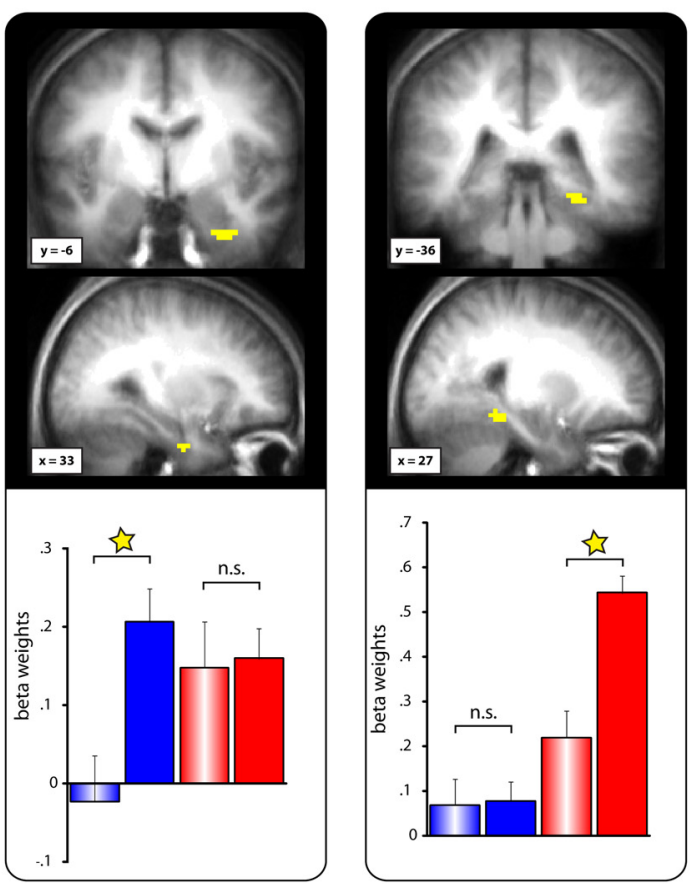

B Domain-General Source Encoding Object \& Scene Trials

Hippocampus mid-PhG
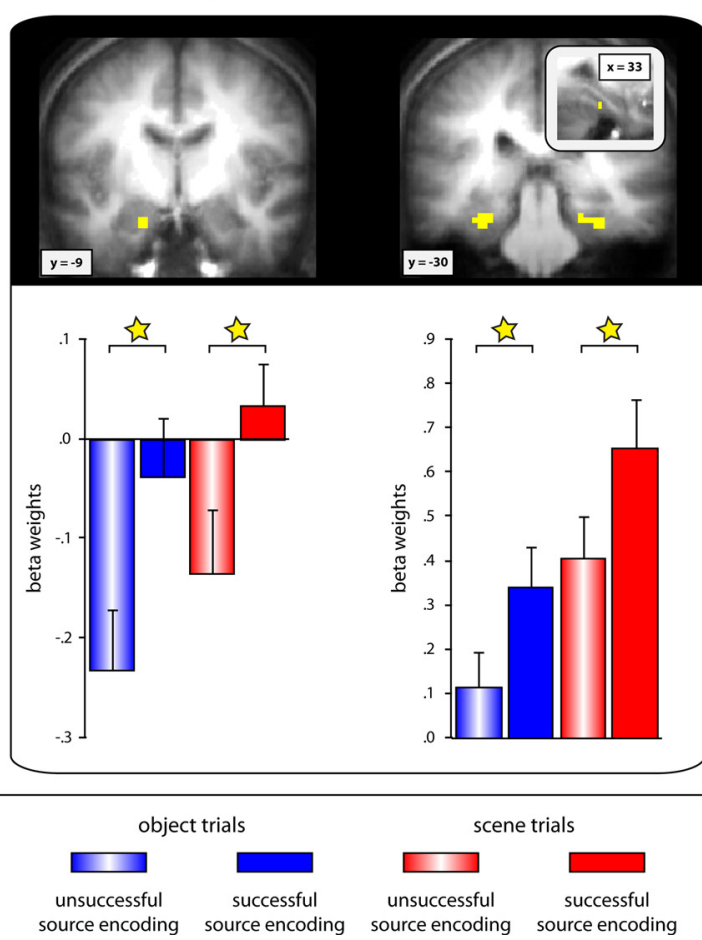

Figure 3. Domain-specific $(\boldsymbol{A})$ and domain-general $(\boldsymbol{B})$ source encoding effects in the medial temporal lobe revealed via contrast/masking procedures within the standard GLM approach. Clusters are displayed on the mean anatomical scan across subjects. The bar graphs represent mean ( + SEM) $\beta$-weights across subjects (averaged across voxels within a cluster) for successful (filled bars) and unsuccessful (striped bars) object (blue) and scene (red) source encoding trials. ${ }^{3} p<0.05 ;$ n.s., not significant. was confirmed via a significant region by domain interaction $\left(F_{(1,18)}=21.82 ; p<0.001\right)$. In sum, these results rule out the possibility that the effects we observed in $\mathrm{PrC}$ and $\mathrm{PhC}$ merely reflect successful mental imagery and point to a direct role in successful source encoding.

\section{Task effects}

Finally, given that the target stimulus during encoding (i.e., the adjective) was held perceptually constant and by itself did not reflect different representational domains, a critical question was whether subjects indeed engaged in domain-specific mental imagery. Although the fact that source memory was clearly above chance suggests that subjects were able to successfully separate object from scene trials during the encoding phase, we queried our imaging data for additional evidence for object- and scene-specific imagery. We reasoned that regions known to be preferentially involved in spatial/scene processing, such as the "parahippocampal place area (PPA)" (Epstein and Kanwisher, 1998), the retrosplenial cortex (RSc) (Vann et al., 2009), or the hippocampus (Bird and Burgess, 2008), should be more strongly engaged during scene compared with object trials, whereas the inverse should be the case for regions preferentially involved in object processing such as the "lateral occipital complex (LOC)” (Malach et al., 1995). We thus directly compared, in the context of the main repeated-measures ANOVA $(p<0.001$, uncorrected; minimum of 5 contiguous voxels), all scene trials with all object trials (regardless of subsequent memory performance). Indeed, we found that, whereas scene trials were accompanied by enhanced activation in PPA, RSc, and the hippocampus relative to object trials, the inverse was true in LOC (supplemental Fig. S4, available at www.jneurosci.org as supplemental material). Given our restricted brain coverage (our slice prescription yielded a limited field of view, not covering lateral parietal and inferior frontal regions), this analysis should not be considered conclusive, but the dissociation between object and scene trials in canonical object- and scene-processing "modules" further suggests that subjects successfully engaged in domain-specific imagery. In other words, although the perceptual input remained constant, our data indicate that subjects successfully produced domain-specific cognitive representations during object and scene imagery.

\section{Discussion}

This study was designed to elucidate the roles of medial temporal lobe cortex (MTLC) regions, namely the $\mathrm{PrC}$ and $\mathrm{PhC}$, during successful memory formation. We used a source memory paradigm in which the criterial to-be-remembered episodic detail was either object or scene related (Fig. 1), but the perceptual input was held constant. Our data revealed a double dissociation between $\mathrm{PrC}$ and $\mathrm{PhC}$ during successful encoding of object- versus scene-related source information, respectively (Figs. 2, 3). These results strongly point to representational domain as a key factor modulating the contribution of these regions to episodic memory (Davachi, 2006).

Before discussing the results in more detail, a note on terminology. Throughout the paper, we use "source memory" to denote memory for whether the target adjective was encountered in the object or scene imagery condition. However, the precise mnemonic evidence on which subjects base their response may vary on a subject-by-subject and/or trial-by-trial basis. For instance, it is conceivable that, for example, when seeing the adjective "loud" at test, subjects remember an abstract context ("this was in the object condition"/“I imagined some object"). Alternatively, sub- 
jects might recover a specific object representation they imagined for "loud" (e.g., an amplifier), essentially being cued to recollect a paired-associate object representation. Although we remain agnostic as to which exact strategy was used, we point out that remembering the particular encoding task in which a target item had been encountered has been used as the canonical "source"/ "context"/"recollection" assessment in the recent neuroimaging literature (Davachi et al., 2003; Ranganath et al., 2004; Gold et al., 2006; Kensinger and Schacter, 2006; Dougal et al., 2007), and we thus use "source memory" for consistency here.

\section{Object preference in $\mathrm{PrC}$}

Based on animal studies as well as human neuropsychological and functional imaging work, $\mathrm{PrC}$ processes have been intimately linked with familiarity-based recognition (for review, see Brown and Aggleton, 2001; Eichenbaum et al., 2007; Mayes et al., 2007). Indeed, initial fMRI studies showed that, whereas activation in the hippocampus and $\mathrm{PhC}$ correlated with later context/source memory, activation in $\mathrm{PrC}$ did not correlate with these measures, but instead predicted nonassociative item memory (Davachi et al., 2003; Ranganath et al., 2004; Kensinger and Schacter, 2006; Dougal et al., 2007). However, in a series of recent fMRI studies, we have shown that $\mathrm{PrC}$ encoding processes can support multiple expressions of memory, including simple item as well as associative recognition, as long as the criterial event details defining item or associative recognition are object related (e.g., an object's color) (Staresina and Davachi, 2006, 2008, 2010). These findings suggested that $\mathrm{PrC}$ encoding processes can contribute to later item familiarity as well as recollection of object- or item-related details. It has been argued, however, that the associations supported by $\mathrm{PrC}$ reflect rather inflexible, "unitized" representations, which in turn are more likely to be accompanied by familiarity than by recollection (Haskins et al., 2008; Diana et al., 2010). However, in our current paradigm, we found $\operatorname{PrC}$ source encoding effects when conditionalizing source memory on remembering the associated encoding task, which has been the canonical assessment of recollection- rather than familiaritybased memory (Johnson et al., 1993; Davachi et al., 2003; Ranganath et al., 2004; Kensinger and Schacter, 2006; Dougal et al., 2007). Moreover, to directly quantify whether source memory for object trials was supported by recollection in our paradigm, we conducted a separate behavioral experiment in which we explicitly instructed subjects to differentiate between (1) recollecting specific event details and (2) being highly or moderately confident that an adjective was encountered in one of the two encoding tasks. Intriguingly, results showed that correct source memory for object trials was primarily accompanied by recollection (even more so than source memory for scene trials) (supplemental Fig. S5, available at www.jneurosci.org as supplemental material). Together, although the current experiment was not designed to assess the contribution of $\mathrm{PrC}$ to familiarity-based recognition, the results strongly support the conclusion that $\mathrm{PrC}$ encoding processes contribute to recollection of object-related event details.

Finally, it should be noted that, although PrC encoding activation did not correlate with successful source encoding for scene trials in this paradigm, we are cautious about inferring object specificity rather than object preference in $\mathrm{PrC}$. For instance, a recent fMRI study by Montaldi et al. (2006) showed PrC sensitivity to recognition memory for scene stimuli. Although that study assessed item recognition rather than source memory and reported retrieval rather than encoding data, it suggests that $\operatorname{PrC}$ may contribute to scene-related memory under certain circumstances.

\section{Scene preference in (posterior) $\mathrm{PhC}$}

In contrast to $\mathrm{PrC}$, PhC has consistently been implicated in source/contextual recollection of event details (Davachi, 2006; Diana et al., 2007; Ranganath, 2010). Importantly, source/context memory has hitherto been used in a domain-general sense, in line with results suggesting that $\mathrm{PhC}$ supports memory for both spatial and nonspatial contexts (Bar and Aminoff, 2003; Aminoff et al., 2007). Critically, however, although the data reported by Aminoff et al. (2007) showed spatial as well as nonspatial context effects in a mid-PhG region (labeled anterior $\mathrm{PhC}$ in their report), context effects in posterior portions of $\mathrm{PhG}$ were restricted to spatial information. Our current results fit nicely with this pattern: When segmenting the $\mathrm{PhG}$ into $\mathrm{PrC}$ and $\mathrm{PhC}$ only, $\mathrm{PhC}$ source encoding effects were domain preferential, correlating with later memory for scene but not for object trials. Critically, however, a more fine-grained segmentation of the $\mathrm{PhG}$ revealed that, whereas the posterior portion of PhG (covering $\mathrm{PhC}$ ) still showed scene-preferential source encoding effects, a more anterior PhG region, covering the anatomical transition from anterior $\mathrm{PhC}$ to posterior $\mathrm{PrC}$, showed domain-general source encoding effects (i.e., predicted successful source memory for both object and scene trials). Together, these data suggest that the intermediate area between PrC and PhC may support encoding of both spatial/scene and nonspatial/object associations. In sum, our results are consistent with the notion that $\mathrm{PhC}$ supports source/contextual encoding (Davachi, 2006; Diana et al., 2007; Ranganath, 2010) but importantly highlight differences between anterior (domain-general) and posterior (scene-preferential) $\mathrm{PhC}$ source/context encoding effects.

It deserves explicit mention that we observed the mid-PhG domain-general source encoding effect not only in the standard GLM analysis, but also in the ROI analysis in which we used unnormalized and unsmoothed data. This ensures that the effect is not the result of artifactual spillover from the domainpreferential patterns in anterior and posterior segments of PhG. In terms of the functional architecture of the MTLC, this transitional zone might reflect a gradient in domain-preference along the anterior-posterior extent of the PhG. Similar results have been reported by Litman et al. (2009) in a blocked fMRI study using a perceptual detection (one-back) task, showing a gradual decrease of scene preference from posterior to anterior portions of PhG.

\section{Division of labor in the MTL during episodic memory formation}

In sum, our current results are in agreement with prevailing frameworks postulating that involvement of specific MTL regions modulates the quality, rather than merely the strength of the ensuing memory trace (Brown and Aggleton, 2001; Aggleton and Brown, 2006; Davachi, 2006; Diana et al., 2007; Eichenbaum et al., 2007; Mayes et al., 2007) (but see Squire et al., 2004, 2007). Specifically, the hippocampus appears to be involved in source encoding across multiple representational domains (Fig. 3B), consistent with the idea that this region supports associative memory in a domain-general manner (Prince et al., 2005; Uncapher et al., 2006; Staresina and Davachi, 2008, 2009; Preston et al., 2010) (for review, see Cohen and Eichenbaum, 1993; Davachi, 2006; Konkel and Cohen, 2009). Conversely, encoding mechanisms in the MTLC (in PrC and PhC) appear to be domain preferential, contributing to episodic memory as a function of the stimulus representation needed for memory performance (Davachi, 2006) (Figs. 2, 3; supplemental Figs. S1, S2, available at www. jneurosci.org as supplemental material). 
What are the functional interactions between the hippocampus and MTLC during successful memory formation? With regard to our current paradigm, one conceivable scenario is that the effective binding of the adjective and the associative/contextual details supporting later source memory is accomplished by the hippocampus, drawing on the domainpreferential representations provided by MTLC regions (Jensen and Lisman, 2005). In that sense, the hippocampus might represent a "convergence zone" for the domain-preferential inputs provided by $\mathrm{PrC}$ and $\mathrm{PhC}$. To further investigate the functional dynamics within MTLC and between MTLC and the hippocampus, enhanced temporal resolution, such as provided by electrophysiological studies in primates (Yanike et al., 2009) or by intracranial EEG recordings in human epilepsy patients (Fernández et al., 1999; Fell et al., 2001), would be highly informative. Importantly, however, the current data provide a critical cornerstone for this endeavor by elucidating the functional segregation among different MTL subregions in the service of episodic memory formation.

\section{References}

Aggleton JP, Brown MW (2006) Interleaving brain systems for episodic and recognition memory. Trends Cogn Sci 10:455-463.

Alvarado MC, Bachevalier J (2005) Comparison of the effects of damage to the perirhinal and parahippocampal cortex on transverse patterning and location memory in rhesus macaques. J Neurosci 25:1599-1609.

Aminoff E, Gronau N, Bar M (2007) The parahippocampal cortex mediates spatial and nonspatial associations. Cereb Cortex 17:1493-1503.

Awipi T, Davachi L (2008) Content-specific source encoding in the human medial temporal lobe. J Exp Psychol Learn Mem Cogn 34:769-779.

Bar M, Aminoff E (2003) Cortical analysis of visual context. Neuron 38:347-358.

Bird CM, Burgess N (2008) The hippocampus and memory: insights from spatial processing. Nat Rev Neurosci 9:182-194.

Brett M, Anton JL, Valabregue R, Poline JB (2002) Region of interest analysis using an SPM toolbox. Paper presented at Eighth International Conference on Functional Mapping of the Human Brain, Sendai, Japan, June.

Brown MW, Aggleton JP (2001) Recognition memory: what are the roles of the perirhinal cortex and hippocampus? Nat Rev Neurosci 2:51-61.

Büchel C, Holmes AP, Rees G, Friston KJ (1998) Characterizing stimulusresponse functions using nonlinear regressors in parametric fMRI experiments. Neuroimage 8:140-148.

Cohen NJ, Eichenbaum HE (1993) Memory, amnesia, and the hippocampal system. Cambridge, MA: MIT.

Davachi L (2006) Item, context and relational episodic encoding in humans. Curr Opin Neurobiol 16:693-700.

Davachi L, Mitchell JP, Wagner AD (2003) Multiple routes to memory: distinct medial temporal lobe processes build item and source memories. Proc Natl Acad Sci U S A 100:2157-2162.

Diana RA, Yonelinas AP, Ranganath C (2007) Imaging recollection and familiarity in the medial temporal lobe: a three-component model. Trends Cogn Sci 11:379-386.

Diana RA, Yonelinas AP, Ranganath C (2010) Medial temporal lobe activity during source retrieval reflects information type, not memory strength. J Cogn Neurosci 22:1808-1818.

Dougal S, Phelps EA, Davachi L (2007) The role of medial temporal lobe in item recognition and source recollection of emotional stimuli. Cogn Affect Behav Neurosci 7:233-242.

Eacott MJ, Gaffan EA (2005) The roles of perirhinal cortex, postrhinal cortex, and the fornix in memory for objects, contexts, and events in the rat. Q J Exp Psychol B 58:202-217.

Eacott MJ, Gaffan D, Murray EA (1994) Preserved recognition memory for small sets, and impaired stimulus identification for large sets, following rhinal cortex ablations in monkeys. Eur J Neurosci 6:1466-1478

Eichenbaum H, Yonelinas AP, Ranganath C (2007) The medial temporal lobe and recognition memory. Annu Rev Neurosci 30:123-152.
Epstein R, Kanwisher N (1998) A cortical representation of the local visual environment. Nature 392:598-601.

Fell J, Klaver P, Lehnertz K, Grunwald T, Schaller C, Elger CE, Fernández G (2001) Human memory formation is accompanied by rhinalhippocampal coupling and decoupling. Nat Neurosci 4:1259-1264.

Fernández G, Effern A, Grunwald T, Pezer N, Lehnertz K, Dümpelmann M, Van Roost D, Elger CE (1999) Real-time tracking of memory formation in the human rhinal cortex and hippocampus. Science 285:1582-1585.

Gold JJ, Smith CN, Bayley PJ, Shrager Y, Brewer JB, Stark CE, Hopkins RO, Squire LR (2006) Item memory, source memory, and the medial temporal lobe: concordant findings from fMRI and memory-impaired patients. Proc Natl Acad Sci U S A 103:9351-9356.

Haskins AL, Yonelinas AP, Quamme JR, Ranganath C (2008) Perirhinal cortex supports encoding and familiarity-based recognition of novel associations. Neuron 59:554-560.

Insausti R, Juottonen K, Soininen H, Insausti AM, Partanen K, Vainio P, Laakso MP, Pitkänen A (1998) MR volumetric analysis of the human entorhinal, perirhinal, and temporopolar cortices. AJNR Am J Neuroradiol 19:659-671.

Jacoby LL (1991) A process dissociation framework: separating automatic from intentional uses of memory. J Mem Lang 30:513-541.

Jensen O, Lisman JE (2005) Hippocampal sequence-encoding driven by a cortical multi-item working memory buffer. Trends Neurosci 28:67-72.

Johnson MK, Hashtroudi S, Lindsay DS (1993) Source monitoring. Psychol Bull 114:3-28.

Kensinger EA, Schacter DL (2006) Amygdala activity is associated with the successful encoding of item, but not source, information for positive and negative stimuli. J Neurosci 26:2564-2570.

Konkel A, Cohen NJ (2009) Relational memory and the hippocampus: representations and methods. Front Neurosci 3:166-174.

Litman L, Awipi T, Davachi L (2009) Category-specificity in the human medial temporal lobe cortex. Hippocampus 19:308-319.

Malach R, Reppas JB, Benson RR, Kwong KK, Jiang H, Kennedy WA, Ledden PJ, Brady TJ, Rosen BR, Tootell RB (1995) Object-related activity revealed by functional magnetic resonance imaging in human occipital cortex. Proc Natl Acad Sci U S A 92:8135-8139.

Mandler G (1980) Recognizing: the judgment of previous occurrence. Psychol Rev 87:252-271.

Mayes A, Montaldi D, Migo E (2007) Associative memory and the medial temporal lobes. Trends Cogn Sci 11:126-135.

Meunier M, Bachevalier J, Mishkin M, Murray EA (1993) Effects on visual recognition of combined and separate ablations of the entorhinal and perirhinal cortex in rhesus monkeys. J Neurosci 13:5418-5432.

Montaldi D, Spencer TJ, Roberts N, Mayes AR (2006) The neural system that mediates familiarity memory. Hippocampus 16:504-520.

Pihlajamäki M, Tanila H, Könönen M, Hänninen T, Hämäläinen A, Soininen H, Aronen HJ (2004) Visual presentation of novel objects and new spatial arrangements of objects differentially activates the medial temporal lobe subareas in humans. Eur J Neurosci 19:1939-1949.

Preston AR, Bornstein AM, Hutchinson JB, Gaare ME, Glover GH, Wagner AD (2010) High-resolution fMRI of content-sensitive subsequent memory responses in human medial temporal lobe. J Cogn Neurosci 22:156-173.

Prince SE, Daselaar SM, Cabeza R (2005) Neural correlates of relational memory: successful encoding and retrieval of semantic and perceptual associations. J Neurosci 25:1203-1210.

Pruessner JC, Köhler S, Crane J, Pruessner M, Lord C, Byrne A, Kabani N, Collins DL, Evans AC (2002) Volumetry of temporopolar, perirhinal, entorhinal and parahippocampal cortex from high-resolution MR images: considering the variability of the collateral sulcus. Cereb Cortex 12:1342-1353.

Ranganath C (2010) Binding items and contexts. Curr Dir Psychol Sci 19:131.

Ranganath C, Yonelinas AP, Cohen MX, Dy CJ, Tom SM, D’Esposito M (2004) Dissociable correlates of recollection and familiarity within the medial temporal lobes. Neuropsychologia 42:2-13.

Squire LR, Stark CE, Clark RE (2004) The medial temporal lobe. Annu Rev Neurosci 27:279-306.

Squire LR, Wixted JT, Clark RE (2007) Recognition memory and the medial temporal lobe: a new perspective. Nat Rev Neurosci 8:872-883.

Staresina BP, Davachi L (2006) Differential encoding mechanisms for 
subsequent associative recognition and free recall. J Neurosci 26:9162-9172.

Staresina BP, Davachi L (2008) Selective and shared contributions of the hippocampus and perirhinal cortex to episodic item and associative encoding. J Cogn Neurosci 20:1478-1489.

Staresina BP, Davachi L (2009) Mind the gap: binding experiences across space and time in the human hippocampus. Neuron 63:267-276.

Staresina BP, Davachi L (2010) Object unitization and associative memory formation are supported by distinct brain regions. J Neurosci 30: 9890-9897.

Stark CE, Squire LR (2001) When zero is not zero: the problem of ambiguous baseline conditions in fMRI. Proc Natl Acad Sci U S A 98: $12760-12766$.

Suzuki WA, Amaral DG (1994) Perirhinal and parahippocampal cortices of the macaque monkey: cortical afferents. J Comp Neurol 350: 497-533.

Taylor KJ, Henson RN, Graham KS (2007) Recognition memory for faces and scenes in amnesia: dissociable roles of medial temporal lobe structures. Neuropsychologia 45:2428-2438.

Uncapher MR, Otten LJ, Rugg MD (2006) Episodic encoding is more than the sum of its parts: an fMRI investigation of multifeatural contextual encoding. Neuron 52:547-556.

Vann SD, Aggleton JP, Maguire EA (2009) What does the retrosplenial cortex do? Nat Rev Neurosci 10:792-802.

Wan H, Warburton EC, Kusmierek P, Aggleton JP, Kowalska DM, Brown MW (2001) Fos imaging reveals differential neuronal activation of areas of rat temporal cortex by novel and familiar sounds. Eur J Neurosci 14:118-124.

Worsley KJ, Marrett S, Neelin P, Vandal AC, Friston KJ, Evans AC (1996) A unified statistical approach for determining significant signals in images of cerebral activation. Hum Brain Mapp 4:58-73.

Yanike M, Wirth S, Smith AC, Brown EN, Suzuki WA (2009) Comparison of associative learning-related signals in the macaque perirhinal cortex and hippocampus. Cereb Cortex 19:1064-1078.

Yonelinas A, Jacoby L (1995) The relation between remembering and knowing as bases for recognition: effects of size congruency. J Mem Lang 34:622-643.

Yonelinas AP (2002) The nature of recollection and familiarity: a review of 30 years of research. J Mem Lang 46:441-517. 